\title{
NOUVELLE
}

\section{Virus de l'hépatite C et gouttelettes lipidiques}

Philippe Roingeard, Christophe Hourioux
Inserm ERI 19, Université François Rabelais et CHU de Tours, 10, boulevard Tonnellé, 37032 Tours, France.

roingeard@med.univ-tours.fr

\section{> Le virus de l'hépatite C (VHC)}

Bien que le VHC ait été identifié depuis près de 20 ans et que son génome et ses protéines soient désormais bien connus (Figure 1), le cycle infectieux de ce virus reste encore énigmatique par bien des aspects. Cela résulte principalement de la difficulté, jusqu'à très récemment, de propager le VHC sur des systèmes de culture cellulaire in vitro. Dans ce contexte, différents modèles cellulaires d'étude in vitro ont été mis au point. Parmi ces modèles, les rétrovirus pseudotypés avec les protéines d'enveloppe $\varepsilon 1$ et $\varepsilon 2$ du VHC ont permis d'identifier des éléments clés dans les mécanismes d'entrée du VHC dans la cellule [1]. Les réplicons subgénomiques, constitués par un génome viral contenant une séquence de marqueur de sélection remplaçant la séquence des protéines structurales, ont été un formidable outil pour l'étude de la réplication virale et l'évaluation d'inhibiteurs potentiels de cette réplication [2]. Malheureusement, ces réplicons ne permettaient pas de produire du virus complet et d'étudier la morphogenèse virale. Cette étape du cycle infectieux n'a pu être abordée que grâce à des modèles de surexpression des protéines structurales du VHC, montrant notamment le rôle majeur de la protéine de core dans la morphogenèse de pseudovirions [3, 4]. Finalement, l'année 2005 aura été un tournant dans la recherche sur le VHC, avec la mise au point d'un système cellulaire permettant de reproduire un cycle infectieux complet du VHC in vitro [5]. La communauté internationale des chercheurs sur le VHC doit cette découverte à la persévérance du Dr Takaji Wakita (Tokyo, Japon), qui a eu l'idée de cloner un génome viral à partir du sérum d'un patient atteint d'une hépatite fulminante. Le génome cloné (JFHl) s'est avéré être doté de propriétés particulières (haut niveau de réplication, sans mutations adaptatives en culture cellulaire), permettant la production de particules virales infectieuses. Même si globalement les étapes clés du cycle infectieux (entrée, réplication du génome, morphogenèse) ne sont pas vraiment plus faciles à étudier avec ce

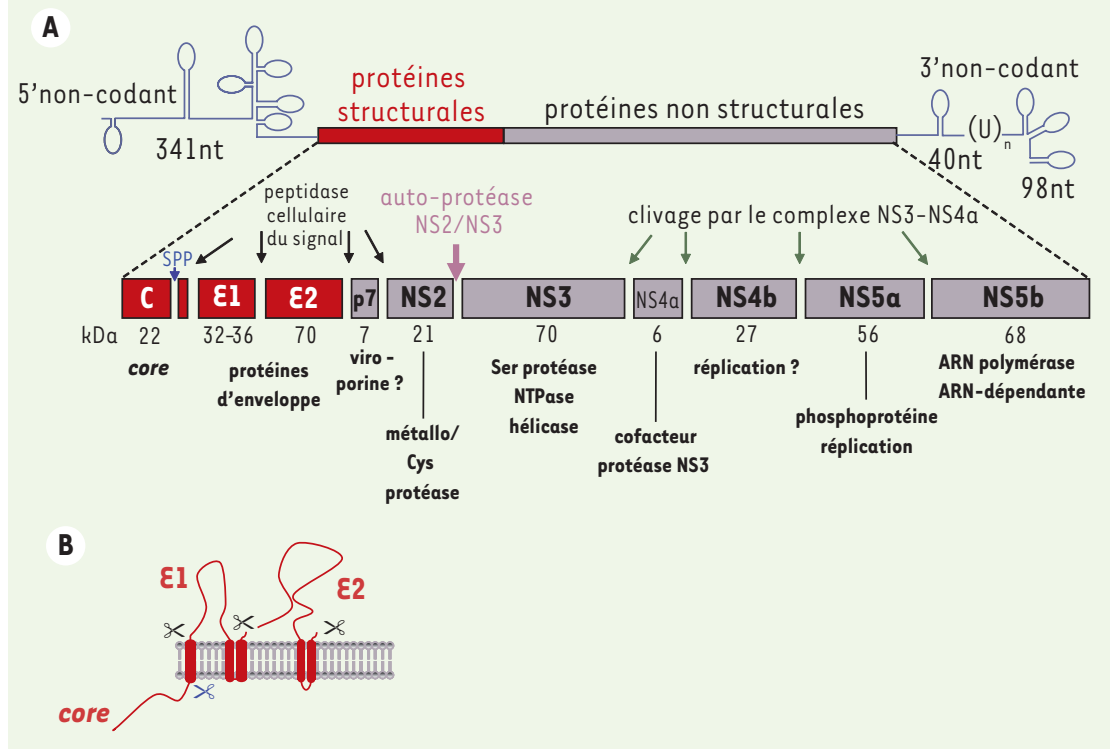

Figure 1. Le génome et les protéines $d u$ VHC. A. Le génome viral consiste en un ARN simple brin positif de 9,6 kb. II code pour une polyprotéine d'environ 3000 acides aminés. Cette région codante est encadrée en 5' par une séquence non codante de 341 nucléotides (nt), contenant un site d'entrée du ribosome (IRES), et en 3' par une séquence non codante essentielle à la réplication virale, de longueur variable. La polyprotéine virale est clivée pour former des protéines structurales (la protéine de core et les deux protéines d'enveloppe $\varepsilon 1$ et દ2), servant à l'assemblage du virus, et les protéines non structurales (de NS2 à NS5b), servant à la maturation de certains domaines de la polyprotéine et à la formation d'un complexe de réplication de l'ARN viral. Ces protéines non-structurales et structurales sont séparées par une petite protéine hydrophobe appelée p7, dont le rôle structural ou non structural n'est pas encore bien précisé. Les protéines structurales sont engendrées par un clivage réalisé entièrement par des peptidases cellulaires présentes au niveau du réticulum endoplasmique $(R \varepsilon)$, alors que les protéines non structurales sont produites par la protéase virale (NS2/NS3). B. Les protéines structurales sont associées à la membrane du RE grâce à une succession de peptides d'initiation et de terminaison de transfert dans la polyprotéine. Sur la face luminale du RE, la peptidase cellulaire du signal libère les protéines d'enveloppe $\varepsilon 1$ et $\varepsilon 2$. La protéine de core subit un clivage additionnel par la peptidase du peptide signal, ou SPP (signal peptide peptidase). 
système qu'avec les modèles alternatifs évoqués ci-dessus, ce modèle a le mérite de reproduire ensemble toutes ces étapes, et avec un virus similaire à ceux qui circulent chez les patients chroniquement infectés.

\section{Les gouttelettes lipidiques}

Ces structures ont longtemps été considérées comme des inclusions cytoplasmiques inertes, ne servant qu'au stockage intracellulaire de triglycérides et d'esters de cholestérol (Figure 2A). Cependant, elles ont connu ces der-

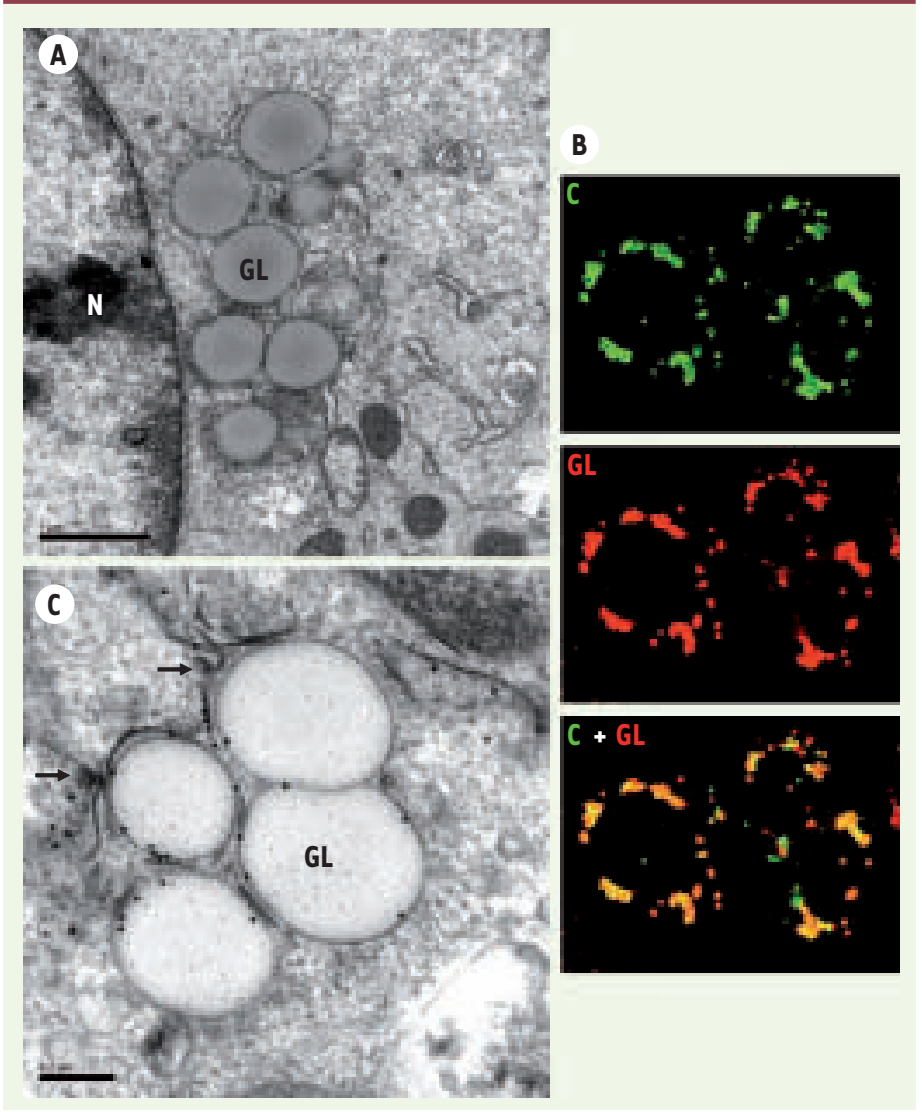

Figure 2. Interaction de la protéine de core du VHC avec les gouttelettes lipidiques. A. En microscopie électronique, les gouttelettes lipidiques (GL) apparaissent comme des structures sphériques homogènes, d'aspect grisé peu dense aux électrons. L'expression de la protéine de core du VHC en système cellulaire in vitro induit leur redistribution autour du noyau (N). B. La microscopie confocale montre la présence de la protéine de core (C) du VHC en surface des gouttelettes lipidiques (GL). C. Des techniques d'immuno-gold utilisant un anticorps monoclonal dirigé contre la protéine de core du VHC montrent la présence de cette protéine à la fois à la surface des gouttelettes lipidiques et dans les membranes du RE adjacentes, au sein desquelles se forment par bourgeonnement les particules virales (flèches). nières années un regain d'intérêt de la part des biologistes cellulaires, pour finalement acquérir un véritable statut d'organite [6]. Participant à la régulation de nombreux mécanismes cellulaires, elles représentent des structures extrêmement mobiles et dynamiques, notamment grâce à des mouvements s'effectuant le long des microtubules du cytosquelette [7]. Outre leur rôle dans la régulation du métabolisme lipidique, les gouttelettes lipidiques seraient aussi responsables des échanges de lipides entre les membranes des

différents compartiments intracelIulaires, contrôlant ainsi la dynamique de la compartimentalisation cellulaire. Elles naissent dans certaines rég i ons du réticulum endoplasmique (RE), par un mécanisme qui n'est pas encorebien déterminé. Un modèle sug g èr e cependant qu'elles pourraient se former par a c cumulation de triglycérides entre les deux monocou- ches de phospholipides de la membrane du RE. Le gonflement de cet espace conduirait alors au bourgeonnement d'une gouttelette entourée d'une monocouche de phospholipides dérivée de la membrane du RE [7]. On ne sait pas cependant si la gouttelette reste associée au RE ou si elle s'en détache complètement pour s'individualiser.

\section{Interaction entre le virus}

\section{et les gouttelettes lipidiques}

Le lien entre le VHC et ces structures cellulaires a été évoqué pour la première fois il y a une dizaine d'années, lorsque des études d'expression de la protéine de core du VHC dans des lignées cellulaires in vitro ont montré que cette protéine se localisait à la surface des gouttelettes lipidiques [8]. Des études complémentaires ont montré que le clivage de la protéine de core par une protéase cellulaire, la SPP (signal peptide peptidase) (Figure IA et $1 B$ ) est nécessaire pour permettre la migration de cette protéine vers la surface des gouttelettes [9]. Après ce clivage, la protéine de core du VHC reste ancrée dans la membrane du RE ou associée aux gouttelettes grâce à la présence d'un domaine carboxy-terminal hydrophobe, sans équivalent dans la protéine de core des autres Flavivirus (à l'exception du virus GBV-B, très proche phylogénétiquement du VHC) [10]. L'expression de la protéine de core du VHC dans des systèmes cellulaires in vitro induit une redistribution des gouttelettes lipidiques dans les zones périnucléaires, associée à une co-localisation entre la protéine et ces gouttelettes (Figure $2 B$ et $2 C$ ). La signification de la localisation de la protéine de core du VHC à la surface des gouttelettes lipidiques n'est pas encore bien comprise. Le modèle des pseudo-virions du VHC a cependant montré que le clivage de la protéine de core par la SPP est non seulement essentiel au trafic de cette protéine vers les gouttelettes, mais est aussi requis pour la formation de la particule virale [11]. En outre, 
ce modèle a permis d'observer que les particules virales s'assemblent et bourgeonnent au niveau de la membrane du $R \varepsilon$, à proximité des gouttelettes lipidiques (Figure 2C). De façon intéressante, dans le système de propagation du virus JFH-1, la redistribution périnucléaire des gouttelettes lipidiques et la localisation de la protéine de core du virus à leur surface est également un événement majeur retrouvé dans les cellules infectées [12], même si ce système ne permet pas de visualiser la morphogenèse du virus. Ainsi, les sites de formation des gouttelettes lipidiques pourraient constituer des microdomaines favorables à l'assemblage des particules virales, en réunissant des facteurs viraux et/ou cellulaires nécessaires à ces mécanismes de morphogenèse, ou en excluant au contraire des facteurs pouvant l'inhiber.

\section{VHC et stéatose hépatique}

Même si l'agression hépatique associée à l'infection chronique par le VHC résulte essentiellement de la réponse immunitaire de l'hôte, certains aspects de la pathogénie viro-induite sont liés à des effets directs du virus. Parmi ces aspects, la stéatose hépatique, caractérisée par l'accumulation de triglycérides dans le cytoplasme des hépatocytes, semble accélérer le développement d'une fibrose du tissu hépatique, qui elle-même contribue à l'installation d'une cirrhose [13]. La protéine de core du VHC peut induire in vitro de multiples effets dans la cellule, en interagissant avec de nombreux partenaires cellulaires modulant l'activité transcriptionnelle ou les voies de signalisation cellulaire. Son impact sur l'apoptose, la transformation cellulaire, le métabolisme lipidique ou la modulation de la réponse immune a fait l'objet de nombreuses études [14]. Toutefois, certains de ces résultats sont contradictoires, la plupart de ces travaux ayant été effectués dans des conditions de surexpression de la protéine, et dans des systèmes qui ne reproduisent pas le cycle infectieux du virus. Concer- nant la modification du métabolisme lipidique, la localisation de la protéine de core du VHC à la surface des gouttelettes lipidiques n'est sans doute pas fortuite, et pourrait avoir un lien avec la survenue d'une stéatose hépatique fréquemment observée chez les patients infectés chroniquement par le VHC. Cette localisation, récemment observée in vitro dans des cellules reproduisant un cycle infectieux complet, conforte cette hypothèse. En clinique, la stéatose hépatique résulte de nombreuses étiologies comprenant un excès de poids, un diabète de type II, une hyperlipidémie, une consommation d'alcool ou la prise de certains médicaments. Cependant, même lorsque toutes ces causes possibles peuvent être exclues, un nombre important de patients atteints d'hépatite chronique $C$ présente une stéatose viro-induite [15]. Même si cette stéatose viro-induite n'est pas spécifique de I'un des six génotypes du VHC, les études cliniques montrent qu'elle est plus fréquente mais également plus sévère chez les patients infectés par un VHC de génotype 3 [15]. Cette observation nous a incité à examiner la variabilité de la protéine de core au sein des génotypes du VHC, tout particulièrement au niveau de son domaine carboxy-terminal hydrophobe exposé après clivage par la SPP. Des investigations conduites à partir de plusieurs centaines de séquences de différents génotypes ont montré que la phénylalanine (F) en position 164 est un résidu spécifiquement retrouvé chez $75 \%$ des VHC de génotype 3 , alors que les autres génotypes possèdent une tyrosine $(y)$ à cette position. Dans un modèle cellulaire in vitro d'expression de la protéine de core du VHC, pour lequel nous avons mis au point une méthode de quantification des gouttelettes lipidiques, il a été montré que l'expression de la protéine de core d'un VHC de génotype 1 induit une augmentation importante de la quantité de gouttelettes lipidiques [16]. De façon tout à fait remarquable, cette même protéine de core, dont le résidu tyrosine en position
164 avait été modifié en phénylalanine (mutant y164F), était capable d'induire une quantité de gouttelettes lipidiques significativement plus importante que la protéine sauvage. Ce modèle cellulaire in vitro reproduit donc bien l'observation d'une stéatose viro-induite et génotype-dépendante évoquée par les études cliniques, et renforce l'idée que l'interaction directe de la protéine de core du VHC avec les gouttelettes lipidiques serait impliquée dans le développement de la stéatose hépatique. Les modèles cellulaires d'étude du VHC pourraient ainsi à terme non seulement permettre de mieux comprendre le cycle infectieux viral et la pathogénie viroinduite, mais également les mécanismes impliqués plus généralement dans la genèse des gouttelettes lipidiques. $\diamond$ Hepatitis C virus and lipid droplets

\section{REMERCIEMENTS}

Nous remercions pour leur soutien l'Agence Nationale de Recherches sur le Sida et les hépatites virales (ANRS), la Ligue contre le Cancer (Comité de l'Indre et Loire et du Cher), la Région Centre (contrat ESPRI, Équipe Soutenue en Partenariat entre la Région et l'Inserm).

\section{RÉFÉRENCES}

1. Bartosch B, Cosset FL. Cell entry of hepatitis C virus. Virology $2006 ; 348: 1-12$.

2. Bartenschlager $R$. The hepatitis $C$ virus replicon system: from basic research to clinical application. J Hepatol $2005 ; 43: 210-6$.

3. Blanchard E, Hourioux C, Brand D, et al. Hepatitis C virus-like particle budding: role of the core protein and importance of its Aspll1. J Virol 2003; 77 : 10131-8.

4. Roingeard $P$, Hourioux C, Blanchard $\varepsilon$, et al. Hepatitis $C$ virus ultrastructure and morphogenesis. Biol Cell $2004 ; 96: 103-8$.

5. Wakita T, Pietschmann T, Kato T, et al. Production of infectious hepatitis $C$ virus in tissue culture from a cloned viral genome. Nat Med 2005 ; 11 : 791-6.

6. Beckman M. Great balls of fat. Science 2006 ; $311: 1232-4$.

7. Martin s, Parton RG. Lipid droplets: a unified view of a dynamic organelle. Nat Rev Mol Cell Biol 2006 ; $7: 373-8$.

8. Barba G, Harper F, Harada T, et al. Hepatitis C virus core protein shows a cytoplasmic localization and associates to cellular lipid storage droplets. Proc Natl Acad Sci USA 1997 ; 94 : 1200-5.

9. McLauchlan J, Lemberg MK, Hope G, Martoglio B. Intramembrane proteolysis promotes trafficking of hepatitis $C$ virus core protein to lipid droplets. EMBO J $2002 ; 21: 3980-8$. 
10. Hourioux C, Ait-Goughoulte M, Patient R, et al. Core protein domains involved in hepatitis $C$ virus-like particle assembly and budding at the endosplasmic reticulum membrane. Cell Microbiol 2007 ; 9: 1014-27.

11. Ait-Goughoulte M, Hourioux C, Patient R, et al. Core protein cleavage by signal peptide peptidase is required for hepatitis C virus-like particle assembly. J Gen Virol $2006 ; 87: 855-60$.
12. Rouillé $Y$, Helle F, Delgrange D, et al. Subcellular localization of hepatitis $C$ virus structural proteins in a cell culture system that efficiently replicates the virus. J Virol $2006 ; 80: 2832-41$.

13. Fartoux F, Chazouillères 0 , Wendum $D$, et al. Impact of steatosis on progression of fibrosis in patients with mold hepatitis C. Hepatology $2005 ; 41: 82-7$.

14. McLauchlan. Properties of the hepatitis $C$ virus core protein: a srtuctural protein that modulates cellular processes. J Viral Hepat 2000 ; $7: 2-14$.

15. Asselah T, Rubbia-Brandt L, Marcellin P, Negro F. Steatosis in chronic hepatitis C: what does it really matter? Gut 2006; 55 : 123-30.

16. Hourioux C, Patient R, Morin A, et al. The genotype 3 -specific hepatitis $C$ virus core protein residue phenylalanine 164 increases steatosis in an in vitro cellular model. Gut 2007 online (sous presse).

\section{NOUVELLE}

\section{Quand les polynucléaires neutrophiles attrapent les agents pathogènes dans leurs filets}

Marie-Anne Gougerot-Pocidalo, Jamel $\varepsilon l$ Benna, Pham My-Chan Dang, Carole Elbim

\author{
Inserm U773, Équipe Phagocytes, NADPH oxydases \\ et Inflammation, Faculté de Médecine, site Bichat, \\ F-75018, Paris, France ; Université Paris 7 Denis Diderot, \\ Faculté de Médecine, site Bichat, Paris, F-75018, France. \\ pocidalo@bichat.inserm.fr
}

> Les polynucléaires neutrophiles humains (PN) constituent l'un des plus puissants systèmes de défense de l'homme contre les agents pathogènes, bactéries et levures, qui ont franchi la barrière cutanéo-muqueuse. Schématiquement, sous l'influence de différents chimioattractants, les PN migrent vers leurs cibles, puis y adhèrent, les englobent et les tuent [1]. Jusqu'à présent, deux mécanismes bactéricides agissant en coopération pouvaient être distingués, l'un dépendant de l'oxygène et l'autre indépendant de celui-ci. (1) Le système tueur indépendant de l'oxygène provient essentiellement du contenu des granulations du neutrophile. Le polynucléaire neutrophile possède en effet au moins trois types de granulations cytoplasmiques: les granulations primaires ou azurophiles, les granulations secondaires ou spécifiques et les vésicules sécrétoires [2]. Lors de la formation du phagosome, les granulations fusionnent avec ce dernier et y déversent de nombreuses substances lytiques et toxiques telles que des hydrolases acides, des défensines ${ }^{1}$, la BPI (bactericidal-perme-

${ }^{1}$ Ce sont des peptides antimicrobiens de nature cationique, non glycosylés et amphiphiliques de 12 à 50 acides aminés. Elles exercent des propriétés antibactériennes, antifongiques et antivirales. Elles sont produites par plusieurs cellules principalement les neutrophiles, les cellules intestinales de Paneth, et les cellules épithéliales. ability increasing protein), l'azurocidine, le lysozyme, la lactoferrine, différentes protéases (élastase, cathepsine G...) qui vont contribuer à la destruction de l'agent pathogène englobé. (2) Le système bactéricide oxygéno-dépendant du polynucléaire neutrophile est puissant et brutal : c'est l'explosion oxydative. Elle est caractérisée par l'activation d'un système moléculaire complexe, la NADPH oxydase, qui induit une réduction de l'oxygène moléculaire en anion superoxyde $\left(\mathrm{O}_{2}^{-} \cdot{ }^{-}\right)$à l'origine d'autres formes réactives de l'oxygène telles que le peroxyde d'hydrogène $\left(\mathrm{H}_{2} \mathrm{O}_{2}\right)$, le radical hydroxyle $\left(\mathrm{OH}^{\circ}\right)$ et l'acide hypochloreux $(\mathrm{HOCl})[3,4]$.

\section{Les filets tueurs des polynucléaires}

Récemment, Brinkmann et al. [5] ont décrit un nouveau mécanisme bactéricide mis en œuvre par les PN : des PN activés par différents stimulus (cytokines, lipopolysaccharide, phorbol myristate acétate) sont capables de former des filaments extracellulaires composés d'ADN, d'histones et de protéines comme l'élastase, la cathepsine $G$, la lactoferrine et la gélatinase, issues des granulations primaires et secondaires (Figure 1 et 2). Les auteurs ont montré que ces filaments appelés NET (neutrophil extracellular traps), ou encore «filets» se lient aux bactéries à Gram positif et à Gram négatif, les piègent et exercent une activité bactéricide extracellulaire à distance sans même les englober (Figure I et 2). Les auteurs montrent que l'élastase localisée au niveau de ces NET dégrade les facteurs de virulence des bactéries pathogènes. La destruction des NET par la désoxyribonucléase, enzyme qui dégrade I'ADN, inhibe leur pouvoir bactéricide. Ces résultats sont interprétés comme un mécanisme de défense innée, indépendant de la phagocytose, qui prévient la dissémination bactérienne ou fungique, permet d'établir des concentrations importantes de substances microbicides dans le voisinage des $\mathrm{PN}$ et de tuer les agents pathogènes. Ce travail n'exclut pas que la formation de ces filets soit une étape très précoce de la mort cellulaire des PN : il est en effet généralement admis que la demi-vie courte de ces derniers est due à une mort rapide soit par nécrose soit par apoptose, selon les conditions de stimulation.

\section{Activité bactéricide}

posthume des polynucléaires

Cette même équipe vient de publier que l'émission de ces NET est associée à la 